\title{
Session 8: Anti-infective, anti-bacterial/viral antibodies: A new emerging paradigm in the field of anti-infectives - I
}

Friday 12 May, 2006. Moderator: Alex von Gabain

[11.00-11.45]

[Keynote Lecture]

A new approach for anti-anthrax mAB

Stanley N Cohen

Stanford University, CA, USA

Abstract not received.

(To be confirmed.)

\section{[11.45-12.05]}

A validated novel genomic approach identifies the antigenomes of pathogens and leads to the selection of protective antigens against major infectious diseases

Eszter Nagy

Intercell AG, Vienna, Austria

The availability of complete genome sequences of pathogens has dramatically changed the perspectives for developing novel vaccines and immune therapies against infectious diseases. Genomics-based strategies and adjunct technologies, including in silico prediction (bioinformatics), expression analyses (random mutagenesis, microarrays, in vivo expression technologies), or protein/peptide based selection methods (proteomics and immunoselection using peptide expression libraries) have already made major contributions to the identification and selection of novel vaccine candidates and antibody targets. Our approach to vaccine development is to learn from individuals who have overcome infectious diseases by the virtue of their own immunity. We have developed a novel technology combining the advantages of full genome coverage and serological antigen identification by using comprehensive smallfragment genomic surface display libraries and antibodies from human serum and body fluids. This method typically selects approximately 150-200 antigenic proteins (the antigenome) and delineates the repertoire of B cell epitopes within ORFs and nonannotated genomic regions. The antigenome technology also offers an integrated approach for epitopebased in vitro antigen validation (without the need for recombinant proteins) that selects the most promising vaccine candidates for animal testing. This comprehensive technology - that can be readily applied to almost all bacterial pathogens and has already been extended to fifteen microbes - has led to the discovery of novel protective antigens from several important human pathogens, including Staphylococcus aureus, Streptococcus pneumoniae, pyogenes and agalactiae. The identified protective antigens are not only candidates for vaccine development, but also good targets for prophylactic and therapeutic mAbs.

\section{[12.05-12.25]}

Therapeutic antibodies: Targeting microbial cell surface adhesin proteins

Joe Patti

Inhibitex Inc., Alpharetta, GA, USA

Abstract not received. 